CASE REPORT

\title{
Parvovirus B19 infection associated with unilateral cervical lymphadenopathy, apoptotic sinus histiocytosis, and prolonged fatigue
}

\author{
T Knösel, H Meisel, A Borgmann, T Riebel, V Krenn, C Schewe, I Petersen
}

J Clin Pathol 2005;58:872-875. doi: 10.1136/icp.2004.022756

This report describes the case of a 16 year old girl with a history of high fever, prolonged fatigue, and cervical lymphadenopathy of the right side. In addition, the patient showed neutropenia, thrombopenia, and pronounced reticulopenia. Cervical ultrasound showed unilateral hypoechoic lymph nodes up to $23 \mathrm{~mm}$ in diameter suspicious for malignant lymphoma. Histology of a cervical lymph node specimen revealed massive nodular histiocytic proliferation and prominent apoptosis without necrosis. Parvovirus B19 was detected by polymerase chain reaction and immunohistochemistry in the lymph node. In summary, this case is an unusual presentation of parvovirus B19 infection. The virus was identified as the potential causative agent of unilateral cervical lymphoma and apoptotic sinus histocytosis, thus broadening the clinicopathological spectrum of parvovirus B19 induced diseases.

W report an unusual presentation of a 16 year old patient with parvovirus B19 infection. The girl was admitted to our hospital with a one week history of high fever and cervical lymphadenopathy. She complained of chronic fatigue over three months, and had been mostly bedridden, unable to visit school. On examination, a tender cervical lymph node with a diameter of $5 \times 8 \mathrm{~cm}$ was palpable in the right cervical region. The left cervical side showed no enlargement. There was no axillary or inguinal lymphadenopathy. She had a rash on both cheeks, which anamnestically occurred intermittently before and after fever periods, so that it could not be clearly attributed to a primary infection.

Cervical ultrasound demonstrated multiple hypoechoic lymph nodes of up to $23 \mathrm{~mm}$ in size, with loss of normal internal structure along the jugular vein, reaching from the right jaw bone angle to the supraclavicular region (fig 1). Haematological studies revealed leucopenia (white blood cell count, $1.8 \times 10^{9} /$ litre), thrombocytopenia (platelets, $127 \times 10^{9} /$ litre), and a normal haemoglobin concentration ( $122 \mathrm{~g} /$ /itre); in addition, erythropoesis was affected by a loss of reticulocytes. Serum analysis revealed a high lactate dehydrogenase value (778 U/litre), suggesting abnormal cell lysis. The erythrocyte sedimentation rate was raised, but $\mathrm{C}$ reactive protein was negative. The humoral immune response parameters were normal (IgG, $13650 \mathrm{mg} / \mathrm{litre}$; IgA, $2940 \mathrm{mg} / \mathrm{litre}$; and IgM, $1430 \mathrm{mg} /$ litre), as were the surface antigen markers of mononuclear cells (CD3, CD4, CD8, CD4/ CD8, CD2, CD16CD56+/CD3-, CD20, CD3/HLA-I, CD4/ CD45RA, CD4/CD45RO, CD3/CD25， CD3/HLA-II， T cell receptor $\gamma / \delta$, CDI4/HLA-II).

The clinical parameters were suspicious for a lymphoproliferative or haemopoetic disease. However, examination of a

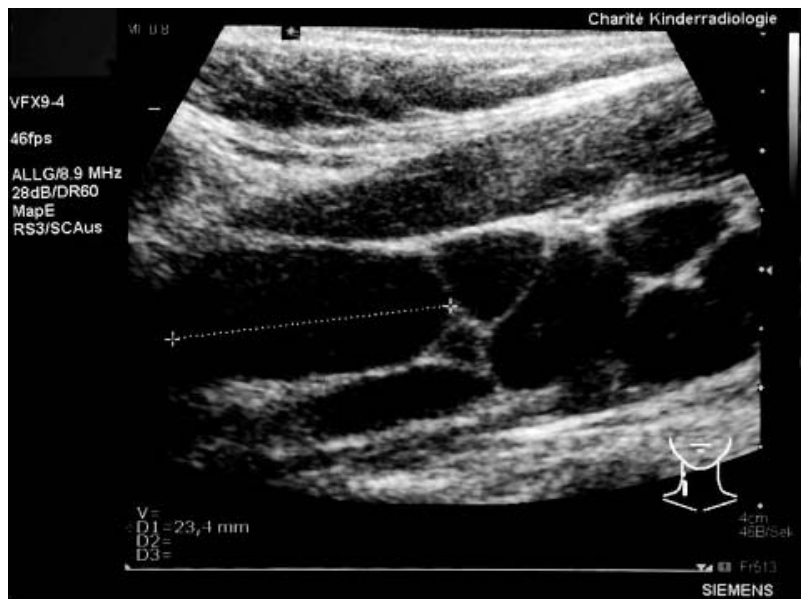

Figure 1 Longitudinal cervical sonogram of the 16 year old girl with prolonged fatigue, demonstrating enlargement of multiple lymph nodes with diffusely reduced echogenicity and loss of typical hilar structures.

bone marrow aspirate showed a hypocellular bone marrow with megakaryocytopenia and reactive lymphocytes compatible with a virus infection. Similarly, the biopsy specimen of a cervical lymph node showed a nodular proliferation of sinus histiocytes with multiple apoptotic bodies without necrosis or infiltration by neutrophils (fig 2A-C).

Although the clinical symptoms were compatible with a systemic ongoing infection, virological studies of the patient's serum on admission for parvovirus B19 (PVB19) proteins revealed negative IgM reactivity against all investigated viral proteins NS1, VP-N, VP-C, VP-1S. NS1 is the non-structural protein 1 of PVB19. VP-N is the $\mathrm{N}$-terminal half and VP-C the C-terminal half of the structural proteins VP1 and VP2, whereas VPIS is a VPl specific segment that is distinct from VP2. In contrast, strong IgG reactivity was seen for VP-N and VP-C, whereas VPIS showed only a weak signal. Serum polymerase chain reaction (PCR) analysis for PVB19 (genotypes 1-3) was consistently negative. There was no serological evidence of Epstein-Barr virus infection.

PCR analysis of the lymph node showed a positive result for PVB19, whereas analysis for other pathogens (EpsteinBarr virus, cytomegalovirus, human herpesvirus 6, human herpesvirus 8, Toxoplasma gondii, Bartonella henselae, and Mycobacterium tuberculosis) was negative. ${ }^{1}$ PVB19 PCR was repeatedly performed by the institutes of pathology and virology of the Charité using different DNA extracts from the lymph node biopsies; the results were mostly positive and PVB19 genotype 1 was revealed.

Abbreviations: $\mathrm{PV}$, parvovirus; $\mathrm{PCR}$, polymerase chain reaction 

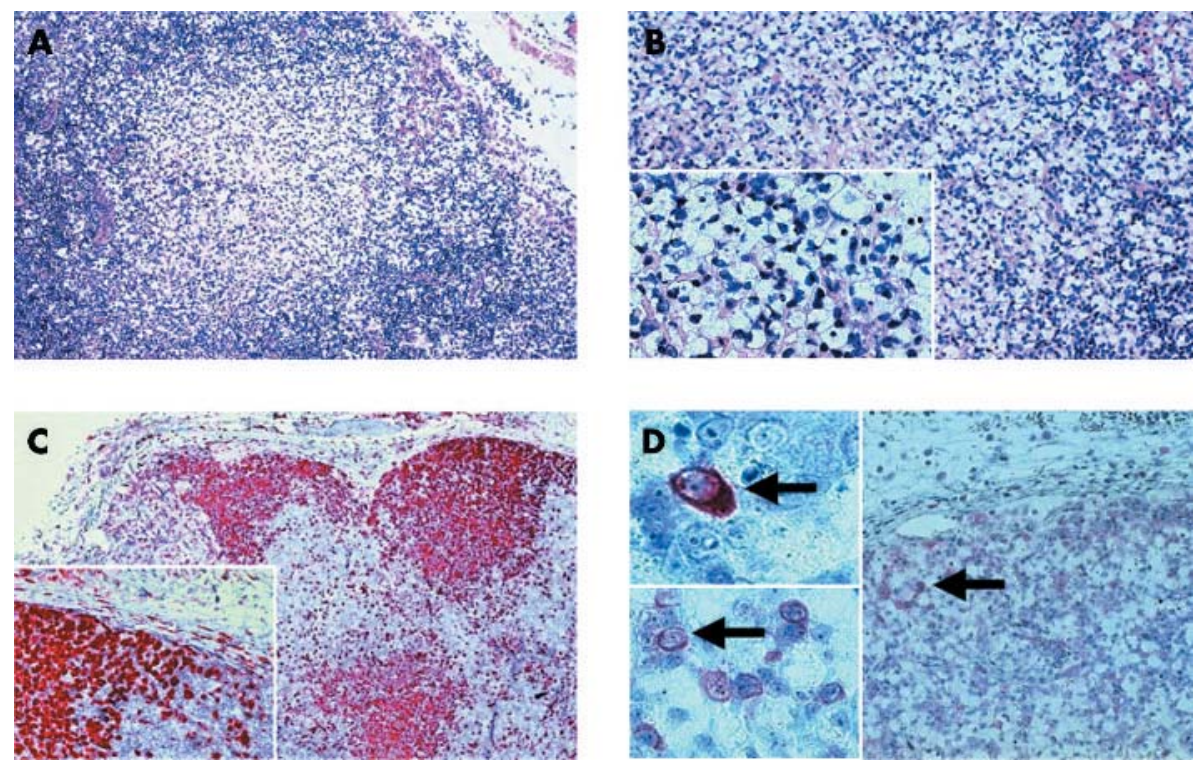

Figure 2 Haematoxylin and eosin (H\&E) and immunohistochemical staining of the lymph node specimen. (A, B) H\&E staining revealed prominent nodules of histiocytes with clear cell cytoplasm and multiple apoptotic bodies (original magnifications: A, x100; B, $\times 200$; insert, $\times 300$ ). (C) The histiocytes were also highlighted by CD68 immunostaining (original magnifications: $\times 150$; insert, $\times 250$ ). (D) Parvovirus B19 immunopositivity was seen in single cells within the lymph node sinuses and parasinal vessels (original magnifications: $\times 150$; inserts, $\times 300$ and $\times 400$; positive cells indicated by arrows).

Immunohistochemical staining for PVB19 structural proteins (VPl and VP2) using the R92F6 antibody ${ }^{2}$ detected sporadic PVB19 reactive cells in the periphery of the histiocytic proliferations and in monocytes of the parasinal blood vessels (fig 2D).

The clinical follow up visit after two months indicated spontaneous remission of the disease.

\section{MATERIALS AND METHODS Detection of PVB 19 DNA}

The following PCR based method was used to detect PVB19 DNA in paraffin wax embedded lymph node tissue. Oligonucleotide primers described by McOmish et al were used to amplify a $219 \mathrm{bp}$ fragment of the viral non-structural protein gene NSPl. ${ }^{3}$ The sequences of the primers were: $5^{\prime}$ GGC AAG AAA AAT ACA CTG (PVI) and 5'-TTG CCC GCC TAA AAT GGC TTT (PV2), position 1390-1608 (NC 000883, AF 162273). The amplification was performed in a $25 \mu \mathrm{l}$ volume using a commercially available reaction mix $(2 \times$ ReddyMix $^{\mathrm{TM}}$, ABgene, Epsom, UK) containing $75 \mathrm{mM}$ Tris/ $\mathrm{HCl}\left(\mathrm{pH} 8.8\right.$ at $\left.25^{\circ} \mathrm{C}\right), 20 \mathrm{mM}$ ammonium sulfate, $1.5 \mathrm{mM}$ $\mathrm{MgCl}_{2}, 0.2 \mathrm{mM}$ of each dNTP, Tween 20, $1.25 \mathrm{U}$ Thermoprime DNA polymerase, loading buffer, and dye for electrophoresis. For the PCR enzyme linked immunosorbent assay (DIG detection; Roche, Penzberg, Germany), a PCR digoxigenin labelling mix (final concentration $120 \mu \mathrm{M}$ ) was added and the primer concentration was adjusted to $0.1 \mu \mathrm{M}$. The amplification was performed as follows: initial denaturation for five minutes at $94^{\circ} \mathrm{C}$, then 40 cycles of one minute at $94^{\circ} \mathrm{C}$, two minutes at $55^{\circ} \mathrm{C}$, and three minutes at $72^{\circ} \mathrm{C}$, followed by seven minutes at $72^{\circ} \mathrm{C}$.

The PCR products were then separated by gel electrophoresis $(3.3 \%$ agarose consisting of two thirds low melting agarose, and one third agarose; Biozym, Wien, Austria) and visualised on an ultraviolet screen after staining with ethidium bromide. A $2.5 \mu \mathrm{l}$ aliquot of the reaction mixture containing digoxigenin labelled PCR products was hybridised with the biotinylated probe PV3 (5'-biotin-ATG GGC CGC CAA GTA CAG GAA A) $)^{3}$ immobilised on a streptavidin coated microtitre plate, and detected as described in the instruction manual for detection of digoxigenin labelled products by a hybridisation based microtitre plate assay (Roche, Mannheim, Germany).

All oligonucleotides were synthesised by BioTez (Berlin, Buch, Germany). Negative control samples (samples without added DNA) were also included in all experiments. A DNA sample from a case that was well characterised microbiologically, immunologically, and clinically served as a positive control. False positive PCR results caused by contamination of samples during all steps of tissue processing were excluded by the inclusion of various preparation and PCR controls. The negative extraction controls were processed in the same manner as the test samples. To demonstrate the presence of amplifiable DNA, all samples underwent a control PCR with the primer pair GH20/PC04, which yields a $268 \mathrm{bp}$ segment of the $\beta$ globin gene. ${ }^{4}$

TaqMan PCR $^{5}$ was used to detect PVB19 in the patient's serum samples. This test is based on the use of the PA-1 and PA-2 primers to amplify the NS1 encoding region of PVB19 genotype 1 and probes $\mathrm{P} 2$ and $\mathrm{P} 3$ to detect genotypes 2 and 3 , respectively.

\section{Detection of PVB 19 antigens}

For immunohistochemistry, $3 \mu \mathrm{m}$ thick, formalin fixed, paraffin wax embedded tissue sections were cut, mounted on slides suitable for use in an automatic immunostaining system (Dako, Glostrup, Denmark), dewaxed with xylene, and gradually hydrated. Antigen retrieval was achieved by pressure cooking in $0.01 \mathrm{M}$ citrate buffer for six minutes. The polyclonal/monoclonal antibody R92F6E (Novocastra Signet Laboratories Inc, Dedham, Massachusetts, USA), which recognises the VP1 and VP2 epitopes, ${ }^{2}$ was diluted 1/40 using a background reducing dilution buffer from Dako. No other blocking agents were used. The primary antibody was incubated at room temperature for one hour. Detection took place by the conventional labelled streptavidin-biotin (LSABkit; Dako) method, with alkaline phosphatase as the reporting enzyme, according to the manufacturer's instructions. Fast red (Sigma, Poole, Dorset, UK) served as the 
Table 1 Symptoms and PVB19 markers in our patient

\begin{tabular}{ll}
\hline Sex & Female \\
Age at onset & 16 years \\
Time since acute PVB19 infection & At least 2 months \\
Deterioration in memory/concentration & No \\
Sore throat & No \\
Tender cervical/axillary lymph nodes & No \\
Painful aching muscles & No \\
Arthralgia & Back pain \\
Arthralgia duration & 3 weeks \\
Joints affected & B and S \\
Joint swelling & No \\
New headaches & No \\
Difficulty sleeping & No \\
Unrefreshing sleep & No \\
Postexertional malaise & Yes \\
Increased tendency to sweat & No \\
Dizzy spells & No \\
Blurred vision or other diseases & No \\
Tender points & No \\
Diagnosis of fatigue & Prolonged fatigue \\
Serum anti-PVB19 NS1, VP-N, VP-C, VP1S lgG & Positive \\
RF & Not tested \\
CRP & Normal \\
ANA & Not tested \\
\end{tabular}

ANA, antineutrophil antibodies; $B$, back; CRP, C reactive protein; PV parvovirus; RF, rheumatoid factor; $S$, shoulders

chromogen. Afterwards, the slides were briefly counterstained with haematoxylin and mounted aqueously.

\section{Detection of PVB 19 antibodies}

Patient serum samples were analysed for PVB19 specific IgM and IgG antibodies against VP2 proteins using enzyme linked immunosorbent assay (Biotrin, Dublin, Ireland) and against the VP1, VP2, and NS1 proteins using western blot analysis (Recom Blot assay; Mikrogen GmbH, Munich, Germany), according to the manufacturer's instructions.

\section{DISCUSSION}

This is the first report of unusual histomorphology in a lymph node biopsy of a patient with PVB19 related prolonged fatigue, pancytopenia, and unilateral cervical lymphadenopathy.

The association between PVB19 and chronic fatigue syndrome has only recently been described.$^{67}$ The fatigue in our patient had been evident for about four months, and should therefore be classified as prolonged fatigue and not chronic fatigue syndrome (longer than six months' duration) according to the criteria established by Fukuda et al. ${ }^{8}$ The clinical and serological parameters of patients with PVB19 associated chronic fatigue syndrome/prolonged fatigue have been well characterised (table 1). ${ }^{7}$ Our patient showed many typical findings. Importantly, other diseases known to be potentially associated with chronic fatigue syndrome-such as rheumatoid arthritis, multiple sclerosis, systemic lupus

\section{Take home messages}

- We report for the first time the histopathology of a patient with parvovirus B19 related prolonged fatigue, pancytopenia, and cervical lymphadenopathy

- This case highlights parvovirus B19 infection as a differential diagnosis in patients with unilateral cervical lymph node swelling

- The histopathological pattern of the parvovirus B19 associated lymphadenopathy was characterised by apoptotic sinus histiocytosis without necrosis erythematosus, and other infectious diseases-could be excluded.

According to the serological parameters, the PVB19 infection occurred at least some months before admission and removal of the lymph node. The time span of the clinical symptoms indicated an acute infection more than three months previously. Together with the negative serum PCR, this suggested a past PVB19 infection. Interestingly, the lymph node pathology, together with the positive detection of the PVB19 genome by PCR and the immunohistochemical detection of the VP1 and VP2 antigens, revealed a locally persistent infection.

\section{"Apoptosis is related to necrosis and may constitute a precursor or an intermediate stage in which the pathogen is unable to cause necrosis"}

The lymph node showed the peculiar morphology of apoptotic sinus histiocytosis without necrosis. Thus, it differed from the pattern of necrotising lymphadenitis (Kikuchi's disease) described so far. ${ }^{9}$ Apoptosis is related to necrosis and may constitute a precursor or an intermediate stage in which the pathogen is unable to cause necrosis. If present, necrosis should lead to alterations of the normal lymph node architecture, such as fibrosis or sclerosis, which were not detectable in our case. The PCR analysis of the lymph node was performed repeatedly, and clearly confirmed the presence of PVB19 genotype 1, suggesting a low viral load. This is consistent with the immunohistochemistry, which revealed only a few infected cells. Taken together, these finding suggest that the apoptotic sinus histiocytosis correlated with a locally persistent infection of PVB19.

The lymph node morphology was distinct from RosaiDorfman disease, which is also characterised by sinus histiocytosis. Typical features of Rosai-Dorfman disease are massive bilateral lymphadenopathy and lymphocytophagocytosis, together with leucocytosis, which were not detectable in our case.

No antiviral or other specific treatment was given and the patient showed no signs of immunodeficiency. Thus, we conclude that the infection was overcome by the patient's immune system.

In summary, this case highlights the association of PVB19 infection and apoptotic sinus histiocytosis in cervical lymphadenopathy in a patient with prolonged fatigue syndrome. The incidence and importance of this finding need to be substantiated by further studies.

\section{Authors' affiliations}

T Knösel, V Krenn, C Schewe, I Petersen, Institute of Pathology, Charité Campus Mitte, 10098 Berlin, FRG

H Meisel, Institute of Virology, Charité - Campus Mitte, 10098 Berlin, FRG

A Borgmann, Department of Paediatric Haematology and Oncology, Charité - Campus Virchow, 13125 Berlin, FRG

T Riebel, Department of Pediatric Radiology, Charite - Campus Virchow, 13125 Berlin, FRG

The patient gave her consent for this report to be published.

Correspondence to: Dr I Petersen, Institute of Pathology, University Hospital Charité, Schumannstrasse 20-21, 10098 Berlin, Germany; iver.petersen@charite.de

Accepted for publication 16 December 2004

\section{REFERENCES}

1 Schewe C, Rizzello M, Dietel M, Hauptmann S. PCR based diagnosis in pathology [review]. Pathologe 2000;21:218-28. 
2 Morey AL, O'Neill HJ, Coyle PV, et al. Immunohistological detection of humanparvovirus B19 in formalin-fixed, paraffin-embedded tissues. J Pathol 1992;166:105-8

3 McOmish F, Yad PL, Jordan A, et al. Detection of parvovirus B19 in donated blood: a model system for screening by polymerase chain reaction. J Clin Microbiol 1993:31:323-8.

4 Saiki RK, Gelfand DH, Stoffel S, et al. Primer-directed enzymatic amplification of DNA with a thermostable DNA polymerase. Science 1988;239:487-91.

5 Liefeldt L, Plentz A, Klempa B, et al. Recurrent high level parvovirus B19/ genotype 2 viremia in a renal transplant recipient analyzed by real-time PCR for simultaneous detection of genotypes 1 to 3. J Med Virol 2005;75:161-9.
6 Matano S, Kinoshita H, Tanigawa K, et al. Acute parvovirus B19 infection mimicking chronic fatigue syndrome. Intern Med 2003;42:903-5.

7 Kerr JR, Bracewell J, Laing I, et al. Chronic fatigue syndrome and arthralgia following parvovirus B19 infection. J Rheumatol 2002;29:595-602.

8 Fukuda K, Straus SE, Hickie I, et al. The chronic fatigue syndrome: a comprehensive approach to its definition and study. International chronic fatigue syndrome study group. Ann Intern Med 1994;121:953-9.

9 Yufu Y, Matsumoto M, Miyamura T, et al. Parvovirus B19-associated haemophagocytic syndrome with lymphadenopathy resembling histiocytic necrotizing lymphadenitis (Kikuchi's disease). Br J Haematol 1997;96:868-71. 